\section{Zero Sero}

\section{Authors: José Barletta}

Submitted:

Published:

Volume:

Issue:

Affiliation:

Languages:

Keywords:

Categories:

DOI:

8

5

English

Medicine
14. November 2021

22. November 2021

El Gato y la Caja, Buenos Aires, Argentina

HIV, Treatment, Patient, Viral suppression, Disease

10.17160/josha.8.5.790

\title{
Abstract:
}

According to UNAIDS, 37.6 million people globally were living with HIV in 2020. In 2016, the United Nations General Assembly's Political Declaration on Ending AIDS established the 90-90-90 targets: (1) to diagnose $90 \%$ of people living with HIV, (2) to secure the treatment for $90 \%$ among people who knew their status, and (3) to reach virally suppression in $90 \%$ of people accessing treatment by the end of 2020 . As Undetectable = Untransmittable, AIDS should be controlled by 2030 . In this article, originally published in El Gato y La Caja, Dr. José Barletta discusses the relevance of viral suppression in HIV+ patients. 


\section{Zero sero}

\section{José Barletta}

Illustrator: Gastón Gonzalez

Date of previous publication: 4/10/19

Link to original note: https://elgatoylacaja.com/cero-sero/

\section{Abstract:}

According to UNAIDS, 37.6 million people globally were living with HIV in 2020. In 2016, the United Nations General Assembly's Political Declaration on Ending AIDS established the 90-90-90 targets: (1) to diagnose $90 \%$ of people living with HIV, (2) to secure the treatment for $90 \%$ among people who knew their status, and (3) to reach virally suppression in $90 \%$ of people accessing treatment by the end of 2020. As Undetectable = Untransmittable, AIDS should be controlled by 2030. In this article, originally published in El Gato y La Caja, Dr. José Barletta discusses the relevance of viral suppression in HIV+ patients.

From a disease of unknown, intractable, and invariably fatal origin, to a chronic and manageable disorder that can be diagnosed with a finger prick and prevented with one pill per day: this is how the last thirty years of the HIVIAIDS epidemic around the world could be summarized.

Despite decades of scientific-technological development, there is one aspect that cannot be overly noted: according to data from the latest Epidemiological Bulletin. the number of new infections remains relatively stable in recent years - the curve is looks like a peak of infections and deaths at the beginning of the epidemic, with a marked decrease after the appearance of highly effective treatments, and a number of new cases that is, as we said, stable: about 6000 people are infected with HIV every year in Argentina; and the incidence is increasing in certain segments of the 
population, such as cis males (i.e., those whose gender assigned at birth coincides with the self-perceived gender) who have sex with other males.

\section{HIV for beginners}

The Human Immunodeficiency Virus (HIV) belongs to the group of retroviruses that mostly affects cells of the immune system, progressively weakening it and allowing 'opportunistic' diseases and infections to appear, which usually do not occur in people whose immune system functions normally. This state of deep immunosuppression that occurs because of HIV is what we know as Acquired Immunodeficiency Syndrome (AIDS). The time that passes from when a person is infected with the virus until disease development is variable, and can range from five to ten years. This process is neither inevitable nor irreversible: antiretroviral treatment works by blocking the replication of the virus and preventing it from damaging the immune system. That is, and this concept is key: being HIV positive is not the same as having AIDS.

HIV can only be transmitted through contact with body fluids that have high concentrations of copies of the virus, such as blood, semen, vaginal and rectal secretions, amniotic fluid or breast milk, among others. While the virus can be isolated in other fluids such as tears, sweat or saliva, the concentration is so low that it has no infective capacity. Therefore, transmission can only occur sexually, bloodily, or perinatally. That is, for having sex without a condom, for sharing unsafe elements for injection, due to puncture accidents or uncontrolled blood transfusions, or from a pregnant person to their son or daughter during pregnancy, childbirth or lactation. But it cannot be transmitted by kisses, hugs, sharing mate or other forms of everyday contact.

\section{One pill per day}

In 1981, the U.S. Center for Disease Control and Prevention (CDC) released a statement warning of "rare cases of pneumonia in 5 gay men in Los Angeles." It took six years until the Food and Drug Administration or FDA (responsible for the regulation of drugs, medical devices and food) approved the use of zidovudine (the famous AZT) for the treatment of HIV infection. AZT had initially been developed for the treatment of cancer, but shelved for its high toxicity and low efficacy in controlling that disease. Approving it as an HIV treatment involved a whole revision: AZT changed the paradigm of the FDA's bureaucratic circuits for diseases of public health 
interest and for which there is - and was not, at the time - anything available. But, even so, it was achieved.

The 'HAART era' (Highly Active Antiretroviral Therapy) began in 1996, a then novel concept presented at the 11th International AIDS Conference in Vancouver. Nowadays, HAART is the role model in the treatment of HIV infection: a combination of different antiretroviral drugs - what is usually called a 'cocktail' with a questionable sense of taste - that is much more effective in controlling the infection than the use of a single drug or monotherapy, which in the 'pre-HAART era' meant standard of care. From then on, the race began to find the combination of antiretrovirals that was not only effective in controlling the infection, but also had the least amount of adverse effects and required fewer pills per day. It was a long way to reach the 'Single Pill Regimens', with which today a person with HIV has an expectation and quality of life equal to that of someone without the virus, taking only one pill a day.

\section{$90-90-90$}

In 2006, the Argentine scientist Julio González Montaner introduced a revolutionary concept: if the probability of transmission is directly related to the number of HIV particles in the blood or 'viral load', and antiretroviral treatment is able to inhibit the replication of the virus to the point where it cannot be detected with conventional analyses (i.e., achieving an 'undetectable viral load'), treating a large number of people with HIV should lower the 'community viral load' and therefore decrease the number of new cases. The idea of Treatment as Prevention (TasP) allowed to universally expand access to antiretroviral therapy (ART) as a strategy capable of improving the expectancy and quality of life of people with HIV, and also of avoiding new infections.

Following this line, the World Health Organization set out in 2013 to achieve an ambitious goal: that by 2020 90\% of people with HIV know their diagnosis, that $90 \%$ of them access antiretroviral therapy in a sustained manner and, in turn, that $90 \%$ of those in treatment maintain an undetectable viral load. Ambitious? A little: reaching this $90-90-90$ would allow nothing less than to control the AIDS epidemic by 2030.

\section{Indetectable $=$ Intransmisible}

The idea that antiretroviral treatment can reduce the risk of HIV transmission arose almost at the beginning of the epidemic: in 1994 a study showed that the use of AZT 
in pregnant cis women and their children was associated with a drastic reduction, although not $100 \%$, in the chances of perinatal transmission. But what about sexual transmission, which today accounts for more than $98 \%$ of cases in our country? The situation is not very different: in serodiscordant couples (in which one of the members has HIV and the other does not), the early onset of HAART reduces the possibility of sexual transmission by more than $90 \%$; and if the person with HIV received antiretroviral treatment long enough to reach an undetectable viral load (in most cases this is achieved within six months of starting treatment), the risk of sexual transmission is reduced to zero. Zero. One can not stress enough how impressive and wonderful that data is. But let the data not cover the forest: this only applies to the sexual transmission of HIV and not to other sexually transmitted infections or the possibility of pregnancy. It never hurts to clarify it.

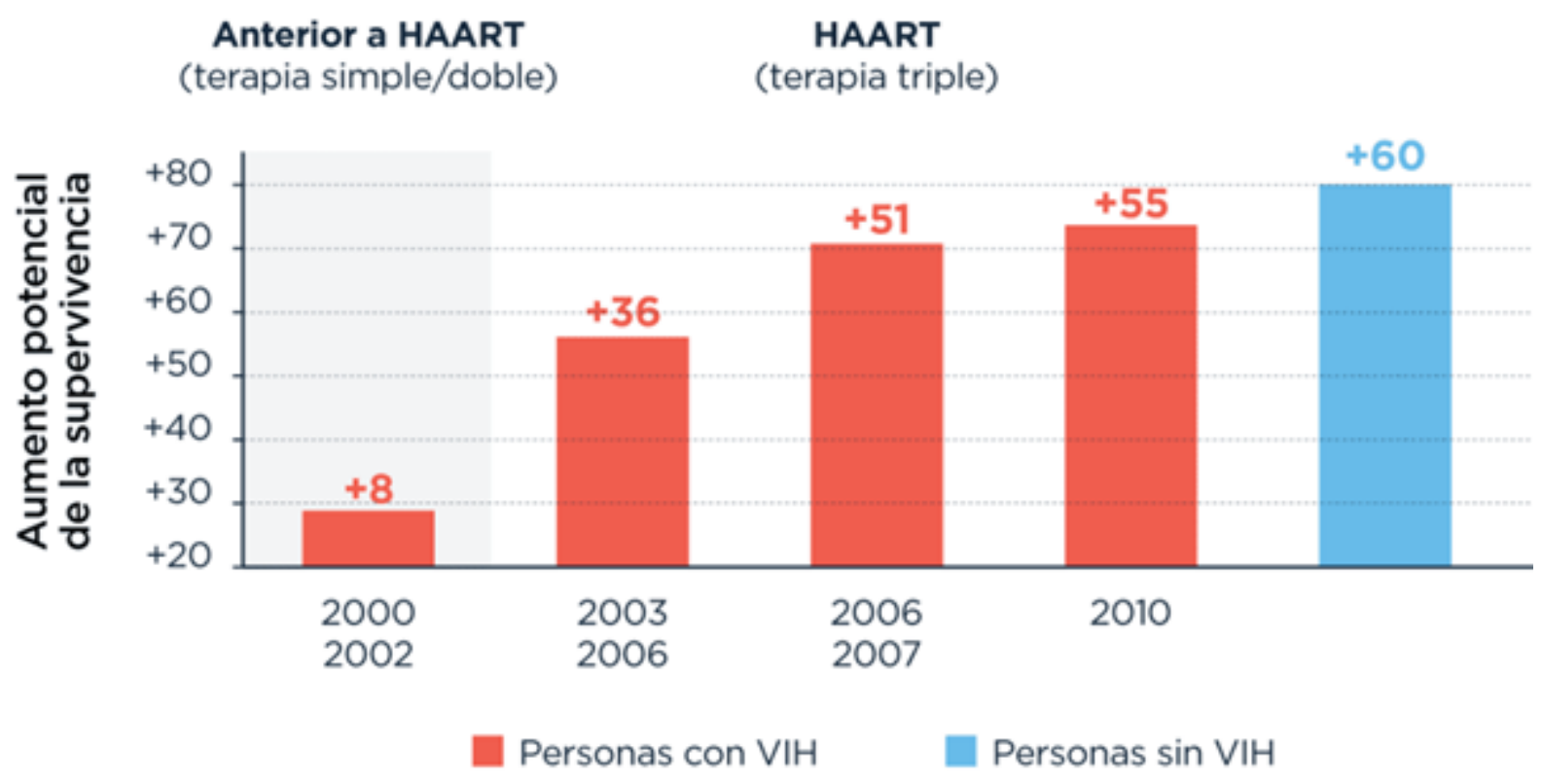

Impact of treatment on the life expectancy of a 20-year-old person with HIV, diagnosed at different times in the history of the epidemic. Source.

The concept of $U=U$ (for Undetectable = Untransmittable) came to change the paradigm and forced to move the focus of attention, to rethink where and in whom we put the effort to reduce the number of new infections, and to get out of our heads the idea that people with HIV who receive treatment are 'source of contagion', 'risk to the environment' and 'responsible for the epidemic': Less than 10\% of HIV cases are diagnosed in the acute stage (i.e. in the period occurring 3 to 4 weeks after 
infection), and it is estimated that up to $70 \%$ of new infections occur from people who contracted the virus recently. This makes sense: in the first days after infection, the viral load is typically very high, the symptoms that occur at this stage in many cases are confused with those of other more frequent minor pathologies such as mononucleosis and - even if the suspicion of the health team is high - during the first two weeks the infection can not be detected by most conventional tests. Hence, most people who are in this acute stage do not know that they are infected.

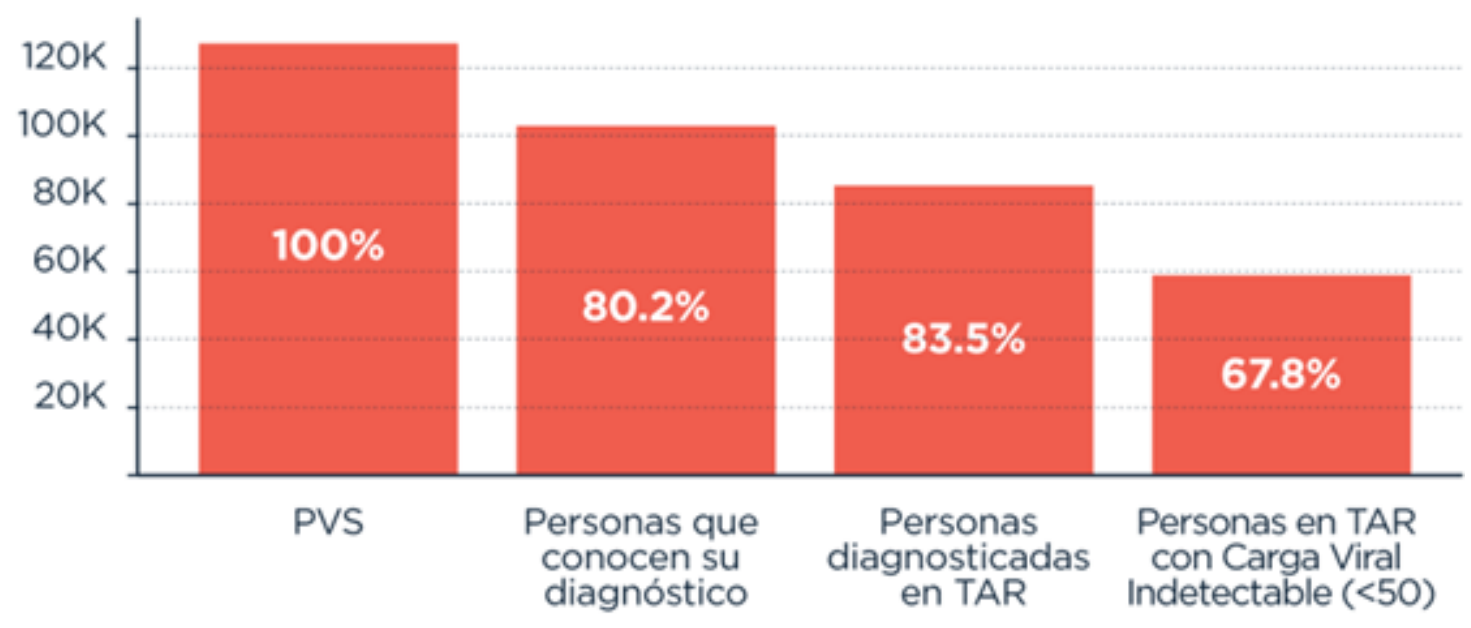

Cascade of the HIV care continuum in Argentina (2017). Source.

\section{Something is not right}

There is effective and - in Argentina - free treatment to control the infection and prevent its transmission, but we have not been able to significantly reduce the number of new cases that occur each year. Without going any further, in Argentina the National AIDS Law (which establishes, among other things, the obligatory nature of informed consent for the performance of the HIV test and the confidentiality of its result) was regulated in 1991 and was a pioneer in the world (something great, although the changes in the epidemic since then stress the need to update it). However, there are still significant and unacceptable disparities and inequities in access to diagnosis and treatment (for example, HIV prevalence among trans women is more than 30 times that of the general population, but their access to the health system is highly conditioned by stigma and discrimination). Something is not right. Or yes. 
Prophylactic antibiotics before surgery to prevent complications, antimalarials to prevent malaria (disease caused by a parasite that is transmitted by mosquitoes) in travelers to endemic areas, eye drops to prevent neonatal conjunctivitis in newborns: the use of drugs to prevent diseases is not a new concept in medicine. In the case of HIV either: the temporary use of antiretrovirals in sons and daughters of people with HIV after childbirth or after sporadic exposure (e.g. a stabbing accident or unprotected sex) is recommended as a standard of care to reduce the risk of infection; and should be available in any public hospital and given free of charge to anyone who has an indication to receive it.

In addition to Post-Exposure Prophylaxis (PEP), there is PrEP or Pre-Exposure Prophylaxis which refers to the use of antiretroviral drugs (typically two, versus the three used to treat people who have HIV) before potential exposure to HIV and generally on an ongoing basis.

The scientific evidence is strong: when taken daily, PrEP is close to $99 \%$ effective in reducing the risk of HIV infection without significant adverse effects. Its use in key populations as part of a combined prevention strategy (which also includes the promotion of condom use, testing for HIV and other STIs, universal access to antiretroviral reatment and comprehensive sex education, among other components) can have a very significant impact: a study conducted in Peru showed that a coverage of only $5 \%$ in populations with high-risk practices could prevent $8 \%$ of new infections.

There are effective and safe drugs to treat it and prevent its transmission. There are accurate and simple methods to diagnose it. There are medications that can prevent it in people at high risk of exposure. There is a scientific community working in the search for a medium-term cure. There are civil organizations that have been conquering rights in terms of access to treatment and elimination of stigma and discrimination for thirty years. Yet, the numbers remain stable. Is it because HIV is a sexually transmitted infection? Would we ask ourselves so many questions before its implementation as a universal public health policy if, instead of preventing HIV in people who do not use PrEP condoms, it reduces by $99 \%$ the risk of cardiovascular disease in sedentary people or lung cancer in smokers? After decades of activism, HIV is on the agenda of many countries. Is taking the remaining steps to achieve control of the epidemic a matter of political commitment? 


\section{References}

https://www.nejm.org/doi/full/10.1056/NEJMoa1600693

https://www.preventionaccess.org/undetectable

http://www.msal.gob.ar/images/stories/bes/graficos/0000001385cnt-2018-12-20_bole tin-epidemiologico-vih-sida-its_n35.pdf 